\title{
Interference Cancellation Schemes for MIMO Multi-carrier CDM in Fading Channels
}

\author{
Rui Liu, Pei Xiao, Jaafar Elmirghani, Colin Cowan
}

A novel interference cancellation (IC) scheme for MIMO Multi-carrier CDM systems is proposed in this correspondence. We show that the existing IC schemes are sub-optimum and their performance can be improved by utilizing some special properties of the residual interference after interference cancellation.

Introduction and System Model: In order to exploit additional frequency and/or time diversity, MIMOOFDM can be combined with Multi-carrier Code Division Multiplexing (MC-CDM) [1], where the signal is spread over multiple OFDM subcarriers using othogonal spreading codes. In [2], three types of spreading matrices are considered and it is shown that the Walsh Hadamard (WH) code provides the best performance compared to other alternatives. At the receiver side, the orthogonality between the spreading codes is lost because the sub-carriers are affected by different fading coefficients [3]. The problem is exacerbated in MIMO systems due to the co-antenna interference (CAI) caused by simultaneous transmissions from different transmit antennas. Hence, efficient data detection techniques must be utilized in order to minimize the effects of channel impairments and interference. The optimal maximum likelihood detector or near-optimal sphere decoder [4] has a prohibitive complexity which prevents it from practical implementation. An IC based detection method has been shown to provide a good trade-off between performance and complexity [1], [3]. By using efficient IC techniques, MCCDM outperforms OFDM in terms of bit error rate performance and bandwidth efficiency [1]. In this correspondence, we propose a novel soft-IC (SIC) scheme for the MIMO-MC-CDM system under study. The performance advantage of the proposed scheme over the conventional hard and soft decision based IC schemes is substantiated by computer simulations.

Fig. 1 shows the block diagram of the MIMO-MC-CDM transmitter with $N$ transmit antennas. First, the binary input bit sequence $\mathbf{b}_{i}$ is mapped to complex valued data symbol sequence $\mathbf{d}_{i}$. Each block of $N Q$ consecutive data symbols, denoted by $\mathbf{d}=\left[d_{1}, d_{2}, \ldots, d_{N Q}\right]$, is serial-to-parallel converted and grouped into $N$ data symbol streams, which can be denoted as an $N \times Q$ matrix $\mathbf{D}=\left[\begin{array}{cccc}d_{1}(1) & d_{1}(2) & \ldots & d_{1}(Q) \\ d_{2}(1) & d_{2}(2) & \ldots & d_{2}(Q) \\ \vdots & \vdots & \ddots & \vdots \\ d_{N}(1) & d_{N}(2) & \ldots & d_{N}(Q)\end{array}\right]$, where each element is denoted as $d_{i}(j) \in \Omega$ where $\Omega$ is a finite set or constellation alphabet, and $Q$ is the number of data sub-carriers in one OFDM frame. An $N \times N$ WH spreading matrix $\mathbf{W}$ is subsequently 
utilized to spread and multiplex the modulated data stream. Mathematically, the composite MC-CDM frame is represented as an $N \times Q$ matrix $\mathbf{S}=\mathbf{W D}$. Each row of the data matrix $\mathbf{S}$ is transmitted by an OFDM module that comprises the inverse Fourier transform (IFFT) and cyclic extension of an OFDM symbol as guard interval or cyclic prefix (CP). Finally, the parallel data streams are transmitted by different antennas.

After CP removal, the OFDM symbol is transformed into the frequency domain by the fast Fourier transform (FFT). This is followed by $Q$ detectors operating in a parallel fashion, one for each subcarrier. The symbol estimates at the output of the detectors are demodulated to obtain an estimate of the transmitted bit sequence $\mathbf{b}_{i}$. Since the same detection mechanism is applied to each detection block, thus only the $k$-th detector will be discussed in the sequel.

The received signal at the $k$-th sub-carrier after CP removal and FFT operation can be expressed as $\mathbf{x}(k)=\mathbf{H}(k) \mathbf{W} \mathbf{d}(k)+\mathbf{u}(k)=\mathbf{V}(k) \mathbf{d}(k)+\mathbf{u}(k)$, where $\mathbf{x}(k)=\left[\begin{array}{llll}x_{1}(k) & x_{2}(k) & \ldots & x_{M}(k)\end{array}\right]^{\mathcal{T}}{ }^{1}$ is the received signal vector; $\mathbf{u}(k)=\left[\begin{array}{llll}u_{1}(k) & u_{2}(k) & \ldots & u_{M}(k)\end{array}\right]^{\mathcal{T}}$ denotes the complex additive white Gaussian noise vector with zero mean and covariance matrix $\sigma_{u}^{2} \mathbf{I}_{M}$. The channel matrix $\mathbf{H}(k) \in \mathbb{C}^{M \times N}$ contains the complex channel gains, and can be formed as $\mathbf{H}(k)=\left[\begin{array}{cccc}H_{11}(k) & H_{12}(k) & \ldots & H_{1 N}(k) \\ H_{21}(k) & H_{22}(k) & \ldots & H_{2 N}(k) \\ \vdots & \vdots & \ddots & \vdots \\ H_{M 1}(k) & H_{M 2}(k) & \ldots & H_{M N}(k)\end{array}\right]$, where $H_{p q}(k)$ is the frequency response of the channel between the $p$-th receive antenna and the $q$-th transmit antenna at the $k$-th sub-carrier. The transmitted symbol vector $\mathbf{d}(k)=\left[\begin{array}{lll}d_{1}(k) & \ldots & d_{N}(k)\end{array}\right]^{\mathcal{T}}$ is the $k$-th column of the matrix $\mathbf{D}$. The data symbols are assumed to be uncorrelated and to have zero mean and unit energy, i.e., $\mathrm{E}\left[\mathbf{d}(k) \mathbf{d}^{\mathcal{H}}(k)\right]=\mathbf{I}_{N}$. The equivalent channel matrix is defined as $\mathbf{V}(k)=\mathbf{H}(k) \mathbf{W}$. The index $k$ will be omitted in the sequel to simplify the notations.

Now let us see how the symbol $d_{n}, n=1, \ldots, N$ can be decoded. After performing parallel interference cancellation, the interference canceled version of the received vector is given as $\mathbf{x}_{n}=\mathbf{x}-\mathbf{V} \overline{\mathbf{d}}_{n}=$ $\mathbf{V}\left[\mathbf{d}-\overline{\mathbf{d}}_{n}\right]+\mathbf{u} \in \mathbb{C}^{N \times 1}$, where $\overline{\mathbf{d}}_{n}=\left[\begin{array}{lllllll}\bar{d}_{1} & \ldots & \bar{d}_{n-1} & 0 & \bar{d}_{n+1} & \ldots & \bar{d}_{N}\end{array}\right]^{\mathcal{T}}$ contains the soft estimate of the interference symbols from the previous iteration, i.e., $\bar{d}_{j}=\mathrm{E}\left[d_{j}\right]$.

In order to further suppress the residual interference in $\mathbf{x}_{n}$, an instantaneous linear filter is applied to $\mathbf{x}_{n}$, to obtain $z_{n}=\mathbf{m}_{n}^{\mathcal{H}} \mathbf{x}_{n}$, where the filter coefficient vector $\mathbf{m}_{n} \in \mathbb{C}^{N \times 1}$ is chosen by minimizing $e_{n}=\mathrm{E}\left\{\left|\mathbf{m}_{n}^{\mathcal{H}} \mathbf{x}_{n}-d_{n}\right|^{2}\right\}$ under the minimum mean square error (MMSE) criterion, and can be derived

\footnotetext{
${ }^{1} M$ is the number of receive antennas. Notations: $(\cdot)^{\mathcal{T}}$ denotes matrix transpose, $(\cdot)^{\mathcal{H}}$ matrix conjugate transpose, $(\cdot)^{*}$ matrix conjugate, $\mathrm{E}[\cdot]$ expectation, and $\mathbf{I}_{N}$ an $N \times N$ identity matrix.
} 
as [5], [6] $\mathbf{m}_{n}=\left[\mathbf{V} \boldsymbol{\Lambda}_{n} \mathbf{V}^{\mathcal{H}}+\sigma_{u}^{2} \mathbf{I}\right]^{-1} \mathbf{v}_{n}$, where the matrix $\boldsymbol{\Lambda}_{n} \in \mathbb{R}^{N \times 1}$ is formed as

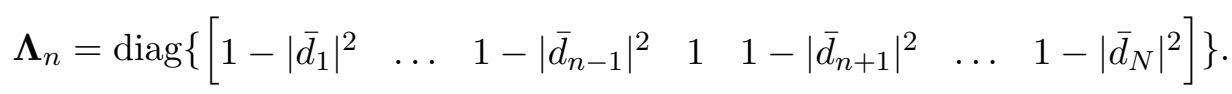

Refer to [5], [6] for more detailed description of the conventional soft IC scheme.

Proposed soft IC scheme: The second-order statistics of a complex random vector $\mathbf{x}_{n}$ are completely characterized by its autocorrelation matrix $\mathbf{C}_{\mathbf{x x}}=\mathrm{E}\left[\mathbf{x}_{n} \mathbf{x}_{n}^{\mathcal{H}}\right]$ as well as the pseudo-autocorrelation matrix $\tilde{\mathbf{C}}_{\mathbf{x x}}=\mathrm{E}\left[\mathbf{x}_{n} \mathbf{x}_{n}^{\mathcal{T}}\right]$. Only the autocorrelation matrix is used in the conventional scheme. While this is the optimum strategy when dealing with circular complex random processes (i.e., when $\tilde{\mathbf{C}}_{\mathbf{x x}}$ is vanishing), it turns out to be sub-optimum in situations where the transmitted signals and/or interference are noncircular random processes (i.e., $\tilde{\mathbf{C}}_{\mathbf{x x}}$ is non-vanishing). It was shown in [7], [8] that the performance of the blind multiuser detectors can be improved by utilizing $\tilde{\mathbf{C}}_{\mathbf{x x}}$. In the sequel, we show how $\tilde{\mathbf{C}}_{\mathbf{x x}}$ can be incorporated into the IC scheme to improve the system performance. To this end, we re-define the MMSE criterion as follows

$$
\epsilon_{n}=\mathrm{E}\left[\left|z_{n}-d_{n}\right|^{2}\right]=\mathrm{E}\left[\left|\boldsymbol{w}_{n}^{\mathcal{H}} \mathbf{y}_{n}-d_{n}\right|^{2}\right]=\boldsymbol{w}_{n}^{\mathcal{H}} \mathbf{C}_{\mathbf{y y}} \boldsymbol{w}_{n}-\boldsymbol{w}_{n}^{\mathcal{H}} \mathbf{C}_{\mathbf{y} d}-\mathbf{C}_{\mathbf{y} d}^{\mathcal{H}} \boldsymbol{w}_{n}+1
$$

where $z_{n}=\boldsymbol{w}_{n}^{\mathcal{H}} \mathbf{y}_{n} ; \mathbf{y}_{n}=\left[\begin{array}{ll}\mathbf{x}_{n} & \mathbf{x}_{n}^{*}\end{array}\right]^{\mathcal{T}} ; \mathbf{C}_{\mathbf{y y}}=\mathrm{E}\left\{\mathbf{y}_{n} \mathbf{y}_{n}^{\mathcal{H}}\right\}=\mathrm{E}\left\{\left[\begin{array}{l}\mathbf{x}_{n} \\ \mathbf{x}_{n}^{*}\end{array}\right]\left[\begin{array}{ll}\mathbf{x}_{n}^{\mathcal{H}} & \mathbf{x}_{n}^{\mathcal{T}}\end{array}\right]\right\}=\left[\begin{array}{ll}\mathbf{C}_{\mathbf{x x}} & \tilde{\mathbf{C}}_{\mathbf{x x}} \\ \tilde{\mathbf{C}}_{\mathbf{x x}}^{*} & \mathbf{C}_{\mathbf{x x}}^{*}\end{array}\right]$, and $\mathbf{C}_{\mathbf{y} d}=\mathrm{E}\left\{\left[\begin{array}{ll}\mathbf{x}_{n} & \mathbf{x}_{n}^{*}\end{array}\right]^{\mathcal{T}} d_{n}^{*}\right\}=\left[\begin{array}{ll}\mathbf{v}_{n} & \mathbf{0}\end{array}\right]^{\mathcal{T}}$. The matrices $\mathbf{C}_{\mathbf{x x}}$ and $\tilde{\mathbf{C}}_{\mathbf{x x}}$ can be computed as $\mathbf{C}_{\mathbf{x x}}=$ $\mathbf{V} \boldsymbol{\Lambda}_{n} \mathbf{V}^{\mathcal{H}}+\sigma_{u}^{2} \mathbf{I}$ and $\tilde{\mathbf{C}}_{\mathbf{x x}}=\mathbf{V} \tilde{\boldsymbol{\Lambda}}_{n} \mathbf{V}^{\mathcal{T}}$, respectively, where

$\tilde{\boldsymbol{\Lambda}}_{n}=\mathrm{E}\left\{\left(\mathbf{d}_{n}-\overline{\mathbf{d}}_{n}\right)^{2}\right\}=\operatorname{diag}\left\{\begin{array}{lllllll}\Lambda_{1} & \ldots & \Lambda_{n-1} & 0 & \Lambda_{n+1} & \ldots & \Lambda_{N}\end{array}\right\}$. Denoting the complex variables $d_{p}=d_{p, I}+j d_{p, Q}$, and $\bar{d}_{p}=\bar{d}_{p, I}+j \bar{d}_{p, Q}$, the $p$-th diagonal element of $\tilde{\boldsymbol{\Lambda}}_{n}$ is calculated as $\Lambda_{p}=\mathrm{E}\left[\left(d_{p}-\right.\right.$ $\left.\left.\bar{d}_{p}\right)^{2}\right]=\mathrm{E}\left[d_{p}^{2}\right]-\left(\bar{d}_{p}\right)^{2}=\mathrm{E}\left[d_{p, I}^{2}+2 j d_{p, I} d_{p, Q}-d_{p, Q}^{2}\right]-\left(\bar{d}_{p, I}\right)^{2}-2 j \bar{d}_{p, I} \bar{d}_{p, Q}+\left(\bar{d}_{p, Q}\right)^{2}=\left(\bar{d}_{p, Q}\right)^{2}-\left(\bar{d}_{p, I}\right)^{2}$. Differentiating $\epsilon_{n}$ in (2) with respect to $\boldsymbol{w}_{n}$ results in $\frac{\partial e}{\partial \boldsymbol{w}_{n}}=\left(\mathbf{C}_{\mathbf{y y}} \boldsymbol{w}_{n}\right)^{*}-\mathbf{C}_{\mathbf{y} d}^{*}$, which is set to zero to yield the optimum vector $\boldsymbol{w}_{n}=\mathbf{C}_{\mathbf{y y}}^{-1} \mathbf{C}_{\mathbf{y} d}$. Note that the conventional filter coefficient vector $\mathbf{m}_{n}$ is calculated using only the autocorrelation matrix $\mathbf{C}_{\mathbf{x x}}$. The pseudo-autocorrelation matrix $\tilde{\mathbf{C}}_{\mathbf{x x}}$ is implicitly assumed to be zero. Omitting $\tilde{\mathbf{C}}_{\mathbf{x x}}$ would consequently lead to sub-optimum solutions.

The MMSE filter output can be expressed as $z_{n}=\mu_{n} d_{n}+\nu_{n} d_{n}^{*}+\eta_{n}$, where the combined noise and residual interference $\eta_{n}$ can be approximated as a Gaussian random variable [5]. The parameters $\mu_{n}, \nu_{n}, N_{\eta}$ can be computed as $\mu_{n}=\mathrm{E}\left\{z_{n} d_{n}^{*}\right\}=\boldsymbol{w}_{n}^{\mathcal{H}}\left[\begin{array}{ll}\mathbf{v}_{n} & \mathbf{0}\end{array}\right]^{\mathcal{T}}, \nu_{n}=\mathrm{E}\left\{z_{n} d_{n}\right\}=\boldsymbol{w}_{n}^{\mathcal{H}}\left[\begin{array}{ll}\mathbf{0} & \mathbf{v}_{n}^{*}\end{array}\right]^{\mathcal{T}}$, $N_{\eta}=\mathrm{E}\left[\left|z_{n}-\mu_{n} d_{n}-\nu_{n} d_{n}^{*}\right|^{2}\right]=\mu_{n}^{*}-\left|\mu_{n}\right|^{2}-\left|\nu_{n}\right|^{2}$. In the derivation of the proposed IC scheme, we take into account the non-circular nature of $\eta_{n}$, and utilize the fact that $\tilde{N}_{\eta}=\mathrm{E}\left[\eta_{n}^{2}\right] \neq 0$, which can be 
computed as $\tilde{N}_{\eta}=\mathrm{E}\left[\left(z_{n}-\mu_{n} d_{n}-\nu_{n} d_{n}^{*}\right)^{2}\right]=\boldsymbol{w}_{n}^{\mathcal{H}} \tilde{\mathbf{C}}_{\mathbf{y y}} \boldsymbol{w}_{n}^{*}-2 \mu_{n} \nu_{n}$. where

$\tilde{\mathbf{C}}_{\mathbf{y y}}=\mathrm{E}\left\{\mathbf{y}_{n} \mathbf{y}_{n}^{\mathcal{T}}\right\}=\mathrm{E}\left\{\left[\begin{array}{l}\mathbf{x}_{n} \\ \mathbf{x}_{n}^{*}\end{array}\right]\left[\begin{array}{ll}\mathbf{x}_{n}^{\mathcal{T}} & \mathbf{x}_{n}^{\mathcal{H}}\end{array}\right]\right\}=\left[\begin{array}{ll}\tilde{\mathbf{C}}_{\mathbf{x x}} & \mathbf{C}_{\mathbf{x x}} \\ \mathbf{C}_{\mathbf{x x}}^{*} & \tilde{\mathbf{C}}_{\mathbf{x x}}^{*}\end{array}\right]=\left[\begin{array}{cc}\mathbf{V} \tilde{\mathbf{\Lambda}}_{n} \mathbf{V}^{\mathcal{T}} & \mathbf{V} \mathbf{\Lambda}_{n} \mathbf{V}^{\mathcal{H}}+\sigma_{u}^{2} \mathbf{I} \\ \mathbf{V}^{*} \boldsymbol{\Lambda}_{n} \mathbf{V}^{\mathcal{T}}+\sigma_{u}^{2} \mathbf{I} & \mathbf{V}^{*} \tilde{\mathbf{\Lambda}}_{n}^{*} \mathbf{V}^{\mathcal{H}}\end{array}\right] ;$

Denote $z_{n}=z_{n, I}+j z_{n, Q}, d_{n}=d_{n, I}+j d_{n, Q}$, and $\eta_{n}=\eta_{n, I}+j \eta_{n, Q}$. To utilize the non-circularity of $\eta_{n}$, we reform the filter output $z_{n}=\mu_{n} d_{n}+\nu_{n} d_{n}^{*}+\eta_{n}$ as $\underbrace{\left[\begin{array}{c}z_{n, I} \\ z_{n, Q}\end{array}\right]}_{\mathbf{z}_{n}}=\underbrace{\left[\begin{array}{c}\left(\mu_{n}+\nu_{n}\right) d_{n, I} \\ \left(\mu_{n}-\nu_{n}\right) d_{n, Q}\end{array}\right]}_{\mathbf{d}_{n}}+\underbrace{\left[\begin{array}{c}\eta_{n, I} \\ \eta_{n, Q}\end{array}\right]}_{\boldsymbol{\eta}_{n}}$. The conditional probability density function (PDF) can then be expressed as

$$
f\left(z_{n} \mid d_{n}\right)=f\left(\mathbf{z}_{n} \mid \mathbf{d}_{n}\right)=\frac{1}{2 \pi \sqrt{\operatorname{det} \boldsymbol{\Sigma}_{n}}} \exp \left(-\frac{1}{2}\left(\mathbf{z}_{n}-\mathbf{d}_{n}\right)^{\mathcal{H}} \boldsymbol{\Sigma}_{n}^{-1}\left(\mathbf{z}_{n}-\mathbf{d}_{n}\right)\right),
$$

where the covariance matrix of the Gaussian noise is $\boldsymbol{\Sigma}_{n}=\mathrm{E}\left[\boldsymbol{\eta}_{n} \boldsymbol{\eta}_{n}^{\mathcal{H}}\right]$. Define a mapping matrix as $\mathbf{J}=\frac{1}{\sqrt{2}}\left[\begin{array}{cc}1 & j \\ 1 & -j\end{array}\right]$, we have $\mathbf{J} \boldsymbol{\Sigma}_{n} \mathbf{J}^{\mathcal{H}}=\mathrm{E}\left[\left(\mathbf{J} \boldsymbol{\eta}_{n}\right)\left(\mathbf{J} \boldsymbol{\eta}_{n}\right)^{\mathcal{H}}\right]=\frac{1}{2} \mathrm{E}\left[\boldsymbol{\epsilon}_{n} \boldsymbol{\epsilon}_{n}^{\mathcal{H}}\right]=\frac{1}{2} \boldsymbol{\Phi}_{n}$, where $\boldsymbol{\epsilon}_{n}=\left[\begin{array}{ll}\eta_{n} & \eta_{n}^{*}\end{array}\right]^{\mathcal{T}}$, and $\boldsymbol{\Phi}_{n}=\mathrm{E}\left[\boldsymbol{\epsilon}_{n} \boldsymbol{\epsilon}_{n}^{\mathcal{H}}\right]=\left[\begin{array}{cc}N_{\eta} & \tilde{N}_{\eta} \\ \tilde{N}_{\eta}^{*} & N_{\eta}\end{array}\right]$. It can be easily shown that $\boldsymbol{\Sigma}_{n}^{-1}=2 \mathbf{J}^{\mathcal{H}} \boldsymbol{\Phi}_{n}^{-1} \mathbf{J}$. The PDF in (3) can thus be reformed as $f\left(z_{n} \mid d_{n}\right)=\frac{1}{2 \pi \sqrt{\operatorname{det} \boldsymbol{\Sigma}_{n}}} \exp \left[-\left(\mathbf{z}_{n}-\mathbf{d}_{n}\right)^{\mathcal{H}} \mathbf{J}^{\mathcal{H}} \boldsymbol{\Phi}_{n}^{-1} \mathbf{J}\left(\mathbf{z}_{n}-\mathbf{d}_{n}\right)\right]$.

In QPSK systems, each symbol $d_{n}$ corresponds to two information bits, denoted as $b_{n}^{0}$ and $b_{n}^{1}$. Denoting the log-likelihood ratio (LLR) value of $d_{n}$ as $\lambda\left(d_{n}\right)=\lambda\left(b_{n}^{0}\right)+j \lambda\left(b_{n}^{1}\right)$, the soft estimate of $d_{n}$ is computed according to its LLR value as $\bar{d}_{n}=\tanh \left[\lambda\left(b_{n}^{0}\right) / 2\right] / \sqrt{2}+j \tanh \left[\lambda\left(b_{n}^{1} / 2\right] / \sqrt{2}\right.$. The LLR value of $b_{n}^{0}$ can then be computed as

$$
\begin{aligned}
\lambda\left(b_{n}^{0}\right) & =\ln \frac{f\left(z_{n} \mid b_{n}^{0}=1\right)}{f\left(z_{n} \mid b_{n}^{0}=0\right)}=\ln \frac{f\left(z_{n} \mid d_{n, I}=+1 / \sqrt{2}\right)}{f\left(z_{n} \mid d_{n, I}=-1 / \sqrt{2}\right)} \approx \ln \frac{\exp \left[-\left(\mathbf{z}_{n}-\mathbf{d}_{+}\right)^{\mathcal{H}} \mathbf{J}^{\mathcal{H}} \boldsymbol{\Phi}_{n}^{-1} \mathbf{J}\left(\mathbf{z}_{n}-\mathbf{d}_{+}\right)\right]}{\exp \left[-\left(\mathbf{z}_{n}-\mathbf{d}_{-}\right)^{\mathcal{H}} \mathbf{J}^{\mathcal{H}} \mathbf{\Phi}_{n}^{-1} \mathbf{J}\left(\mathbf{z}_{n}-\mathbf{d}_{-}\right)\right]} \\
& =\left(\mathbf{z}_{n}-\mathbf{d}_{-}\right)^{\mathcal{H}} \mathbf{J}^{\mathcal{H}} \boldsymbol{\Phi}_{n}^{-1} \mathbf{J}\left(\mathbf{z}_{n}-\mathbf{d}_{-}\right)-\left(\mathbf{z}_{n}-\mathbf{d}_{+}\right)^{\mathcal{H}} \mathbf{J}^{\mathcal{H}} \boldsymbol{\Phi}_{n}^{-1} \mathbf{J}\left(\mathbf{z}_{n}-\mathbf{d}_{+}\right),
\end{aligned}
$$

where $\mathbf{d}_{+}$denotes the vector $\mathbf{d}_{n}$ corresponding to $\max \left\{f\left(\mathbf{z}_{n} \mid d_{n, I}=+1 / \sqrt{2}, d_{n, Q}=+1 / \sqrt{2}\right), f\left(\mathbf{z}_{n} \mid d_{n, I}=\right.\right.$ $\left.\left.+1 / \sqrt{2}, d_{n, Q}=-1 / \sqrt{2}\right)\right\}$, and $\mathbf{d}_{-}$denotes the vector $\mathbf{d}_{n}$ corresponding to $\max \left\{f\left(\mathbf{z}_{n} \mid d_{n, I}=-1 / \sqrt{2}, d_{n, Q}=\right.\right.$ $\left.+1 / \sqrt{2}), f\left(\mathbf{z}_{n} \mid d_{n, I}=-1 / \sqrt{2}, d_{n, Q}=-1 / \sqrt{2}\right)\right\}$. The LLR value of $b_{n}^{1}$ can be computed similarly.

Results: Fig. 2 shows the performance comparison between the different algorithms. By comparison, the proposed SIC detector outperforms the conventional SIC detector by more than $2 \mathrm{~dB}$ at bit error rate of $10^{-4}$. The performance gain gap becomes bigger as SNR increases. The gain is smaller at low SNRs due to the dominance of the circular channel noise. As SNR increases, the performance gain by the proposed detector becomes larger since it benefits more from the exploiting the noncircularity of the interference. The hard decision based IC (HIC) scheme performs much worse than the two SIC schemes. The rationale 
is that HIC is more prone to the error propagation problem, cancellation using incorrect decision will increase interference rather than canceling interference.

Conclusions: A novel interference cancellation scheme for MIMO-MC-CDM systems has been proposed in this correspondence. By exploiting the noncircularity of the interference canceled signal at both the input and output of the MMSE filter, the proposed scheme effectively mitigates the error propagation problem, and achieves superior performance compared with the conventional IC schemes.

\section{REFERENCES}

[1] S. Kaiser. "Performance of multicarrier CDM and COFDM in fading channels", Proc. IEEE Globecom, vol. 1B, pp. 847-851, 1999.

[2] M. Cho, H. Cho, S. Ro, D. Hong. "A novel time spreading method for down-link OFDM-coded division multiplexing systems", Proc. IEEE VTC, pp. 764-768, April 2003.

[3] F. Sanzi. "Comparison of bit error rate and convergence of four different iterative receivers for wireless OFDM-CDM", International Journal of Electronics and Communications, vol. 59, no. 3, pp. 166-176, June 2005.

[4] R. Liu, J. Elmirghani. "Hybrid detectors for wireless OFDM-CDM systems based on reliability information", IET Communications. In press.

[5] X. Wang, H. Poor. "Iterative (turbo) soft interference cancellation and decoding for coded CDMA". IEEE Transactions on Communications, vol. 47, pp. 1046-1061, July 1999.

[6] X. Wautelet, A. Dejonghe, L. Vandendorpe. "MMSE-based fractional turbo receiver for space-time BICM over frequencyselective MIMO fading channels”, IEEE Transactions on Signal Processing, vo. 52, no. 6, pp. 1804-1809, June 2004.

[7] P. Xiao, R. Liu. "Multi-user detector for multi-carrier CDMA systems", IET Electronic Letters, vol. 44, no. 23, pp. 13661368, Nov. 2008.

[8] G. Gelli, L. Paura, A. Ragozini. "Blind widely linear multiuser detection", IEEE Communications Letters, vol. 4, no. 6, pp. 187-189, June 2000 . 


\section{Author's affiliations:}

Rui Liu, Jaafar Elmirghani (School of Electronic \& Electrical Engineering, University of Leeds

LS2 9JT, United Kingdom. E-mail: \{eenrl,j.m.h.elmirghani\}@leeds.ac.uk

Pei Xiao, Colin Cowan (The Institute of Electronics, Communications and Information Technology Queen's University Belfast, BT3 9DT, United Kingdom.

E-mail: \{pei.xiao,c.cowan\}@ecit.qub.ac.uk) 


\section{Figure captions:}

Fig. 1 Block diagram of the MIMO-MC-CDM transmitter.

Fig. 2 Performance comparison of different IC schemes. The curves represent different algorithms at the 3rd iteration when the convergence is reached. Both transmitter and receiver have 4 antennas, i.e., $N=M=4$. A 256-point IFFT/FFT is employed for OFDM demodulation/modulation. A tapped-delayline channel model is used, it has 11 taps with an exponentially decaying power delay profile. The total channel gain is normalized to unity. The length of $\mathrm{CP}$ is set to be $L_{c p}=16$. Perfect knowledge of the channel frequency response is assumed for the investigated IC schemes. For the conventional hard decision based IC (HIC), an MMSE equalizer is employed at the first iteration to obtain an initial estimate of transmitted data. The matched filtering is performed on the interference canceled signals to form a soft decision statistic based upon which a hard decision on the transmitted symbol is made. 


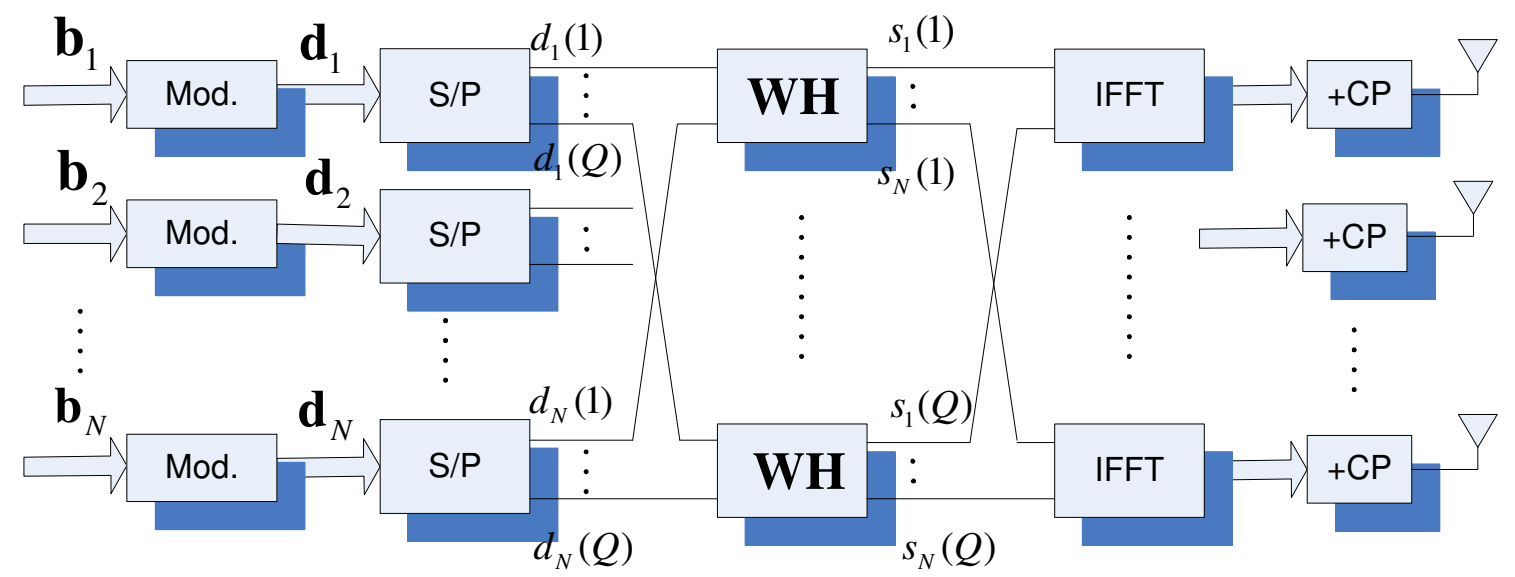

Fig. 1. 


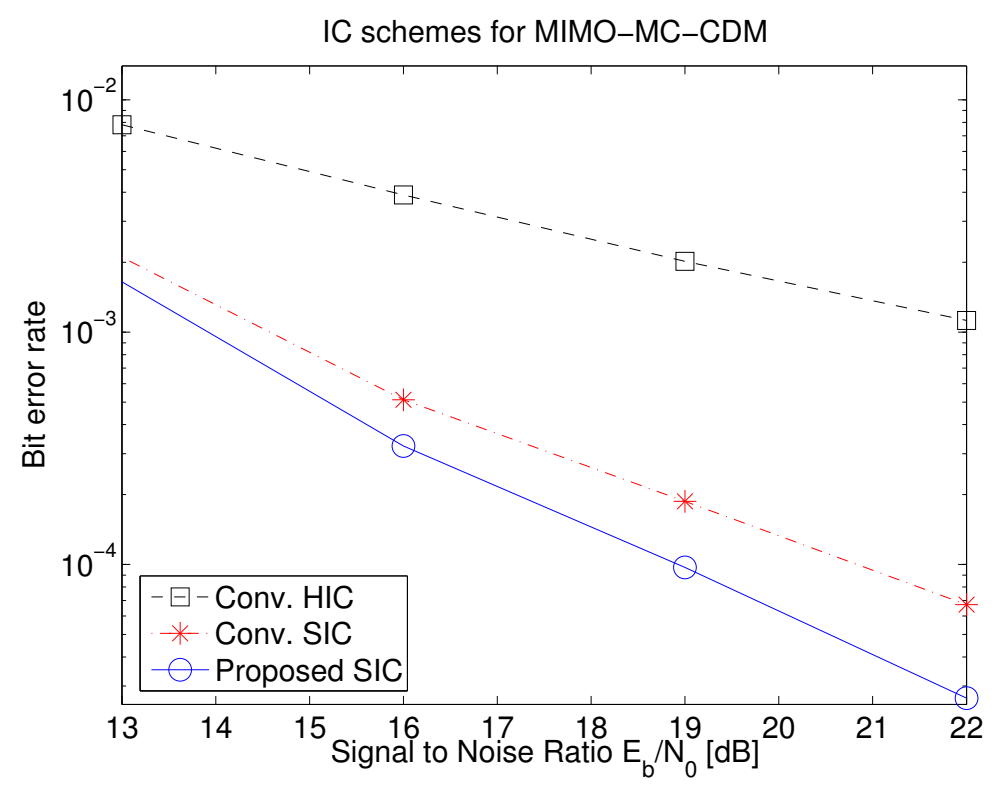

Fig. 2. 